\title{
Perylenediimide-based distributed feedback lasers with holographic relief gratings on dichromated gelatine
}

\author{
Manuel G. Ramírez ${ }^{1,2}$, José A. Quintana1,3, José M. Villalvilla ${ }^{1,2}$, Pedro G. Boj ${ }^{1,3}$, Aritz \\ Retolaza $^{4,5}$, Santos Merino ${ }^{4,5}$ and María A. Díaz-García ${ }^{1,2, a)}$ \\ ${ }^{1}$ Instituto Universitario de Materiales de Alicante (IUMA) and Unidad Asociada UA-CSIC; \\ Universidad de Alicante, 03080- Alicante Spain \\ ${ }^{2}$ Departamento de Física Aplicada; Universidad de Alicante, 03080-Alicante Spain \\ ${ }^{3}$ Departamento de Óptica; Universidad de Alicante, 03080- Alicante Spain \\ ${ }^{4}$ Micro and Nano Fabrication Unit, IK4-Tekniker, Eibar 20600, Spain \\ ${ }^{5}$ CIC microGUNE, Arrasate-Mondragón 20500, Spain
}

\begin{abstract}
One dimensional second-order distributed feedback (DFB) lasers consisting of polystyrene (PS) films doped with a perylenediimide laser dye, deposited over dichromated gelatine (DCG) photoresist layers with solvent resistant relief gratings recorded by holographic lithography, are reported. The advantage of using the grating on DCG in the final device is that the fabrication process is simplified and the grating pattern better preserved, since no etching methods to transfer the grating to another substrate are needed. A very simple model, proposed to explain the experimental waveguide properties, has allowed identifying the waveguide mode at which DFB emission appears, which was the key to optimize the device performance. In the frame of this model, the thickness of the PS and DCG films could be adjusted in order to minimize the laser threshold and to control its wavelength tuneability. The performance of these lasers is comparable to that of recently reported devices based on the same active material, but deposited over high-quality DFB gratings engraved on $\mathrm{SiO}_{2}$ by nanoimprint lithography.
\end{abstract}

\footnotetext{
${ }^{a}$ Author to whom correspondence should be addressed. Electronic mail: maria.diaz@ua.es
} 


\section{INTRODUCTION}

Organic solid-state lasers (OSLs) with active materials in the form of thin waveguide films have attracted great attention in the last two decades due to their easy processability, compatibility with a variety of substrates and the possibility of tuning the optical properties by molecular engineering. ${ }^{1-3}$ Progress achieved in the design and preparation of efficient materials and resonators has led to important improvements in the device performance such as decreasing their laser thresholds, increasing their conversion efficiency, output power and operational lifetimes, or extending their wavelength tuneability range. The demonstration of OSLs pumped with light emitting diodes ${ }^{4}$ has opened the way towards highly integrated organic-inorganic photonics, with a great potential for applications in the fields of optical communications, ${ }^{5}$ biosensing and chemical sensing. ${ }^{2}$

Distributed feedback (DFB) resonators have been widely used to build waveguide-based OSLs. ${ }^{1-3}$ In DFB lasers, feedback is achieved by the incorporation of periodic gratings (obtained by modulating either the refractive index or the gain) that Bragg-scatter the light, thus avoiding the need of good-quality end facets. In addition, these resonators can be easily integrated into planar organic waveguides, which is a clear advantage from the fabrication point of view, as compared to other types of laser cavities. In a one-dimensional (1D) DFB laser, the wavelength that satisfies the Bragg condition $\left(\lambda_{\text {Bragg }}\right)$ given by

$$
m \cdot \lambda_{\text {Bragg }}=2 \cdot n_{\text {eff }} \cdot \Lambda
$$

where $m$ is the order of diffraction, $n_{\text {eff }}$ the effective refractive index of the waveguide, and $\Lambda$ the grating period, constitutes the resonant wavelength in the cavity, which will then be diffracted in the grating in different directions. For second-order DFBs $(m=2$ in eq. (1)) light is coupled out of the film in a direction perpendicular to the waveguide film, by first-order diffraction. 
Various methods for grating fabrication have been used, $^{2}$ including holographic lithography (HL), electron-beam lithography (EBL) and nanoimprint lithography (NIL), among others. The use of HL instead of standard photolithography is because the latter one has limited resolution to achieve the periods required to obtain DFB lasing (i.e. typically between 200 and $500 \mathrm{~nm}$, depending on the material and the diffraction order). NIL has received great attention in the last years for its potential to be scaled to volume production given its high throughput, lowcost and high fidelity pattern transfer. ${ }^{6}$ NIL requires the use of a stamp master, in most cases fabricated by EBL to obtain very high quality patterns, ${ }^{6-9}$ although stamps prepared by other techniques have also been employed. ${ }^{10}$ Recently, our group has reported very efficient DFB lasers based on polystyrene (PS) films doped with perylenediimide (PDI) derivatives as active media, in which high quality $1 \mathrm{D}$ gratings with excellent modulation depth were imprinted by thermal-NIL, either on the substrate ${ }^{7,8}$ or directly on the active film. ${ }^{9}$

The fabrication of DFB lasers using only the HL technique has several advantages with respect to employing NIL, which might be important in certain cases. Firstly, HL provides a great flexibility to modify the grating parameters (dimensionality, period and depth) without the need of a stamp master (with fixed parameters and prepared previously by another method). HL is also an excellent technique when aiming to prepare lasers with large dimensions, i.e. up to a few squared centimeters. Note that the size of the devices prepared by NIL is generally below 1 $\mathrm{mm}^{2}$. It should be noted that in many of the works in which HL has been used to fabricate the DFB resonator, the photoresist layer was not present in the final devices, since gratings were transferred by etching methods to conventional substrates such as glass or $\mathrm{SiO}_{2} \cdot{ }^{1,2,11}$ At this respect, in the last decade many efforts have been devoted to obtain lasers in which the substrate is the photosensitive material over which the grating has been holographically recorded and then, 
the active film is deposited on top of it. This strategy simplifies the fabrication process and consequently the cost of the devices. In addition, the grating pattern is better preserved. Among the photosensitive materials used for that purpose, azo-containing polymers, in which surface relief gratings are recorded by exploiting the photo-isomerization properties of the azocompounds, have been extensively studied. ${ }^{12-15}$ An important milestone recently reported by Goldenberg et al. ${ }^{16}$ consisted of including the active laser dyes within the azobenzene polymer layer over which the DFB grating was recorded, so single layer devices could be obtained. Concerning conventional photoresists, they have been rarely employed in HL as substrates of the final devices. ${ }^{17}$ Recently, photoresists like Ormocore or the epoxy-based negative photoresist SU-8, developed in the middle nineties in the area of photolithography, have been used in the context of more sophisticated technologies, such as combined nanoimprint photolithography (CNP). ${ }^{18-20}$ With respect to the negative photoresist dichromated gelatine (DCG), which has been used in the present work, it has often been employed in classic holography to obtain high efficiency (up to $90 \%$ ) volume gratings by refractive index grating variation. In that case, the light intensity pattern between exposed and unexposed areas is translated in hardness variations and, after a process with water and alcohol, converted in refractive index changes. In fact, the grating in the first DFB laser designed by Kogelnik and Shank ${ }^{21}$ in 1971 was obtained in this way. When DCG is water developed to produce surface-relief gratings, it shows a spatial resolution of less than a few microns. ${ }^{22}$ In 1998, a method to obtain relief gratings with DCG with periods as low as $500 \mathrm{~nm}$ and diffraction efficiencies of $80 \%$ was demonstrated by some of us. $^{23,24}$ The key step to transform hardness variations into relief variations was an anisotropic dry development in an oxygen plasma. Despite the potential of such discovery, its impact has been minor mainly due to some difficulties in handling and processing DCG, together with its low 
sensitivity. Recently, organic DFB lasers consisting of active organic films deposited over corrugated glass substrates with periods of $270 \mathrm{~nm}$, obtained by this technique in combination with reactive ion beam etching, have been demonstrated. ${ }^{11}$

With regards to the active laser material, the selection of an appropriate one is crucial to achieve a good laser performance. The simplest way to assess the laser properties of a given material for waveguide-based laser devices, such as DFBs, consists in studying its amplified spontaneous emission (ASE) properties when deposited as a thin film in a waveguide configuration. ${ }^{1,2,25}$ Typically, the active film is photo-pumped with a pulsed optical source, aiming to identify a collapse of the width of its photoluminescence spectrum and a large enhancement of the output intensity above a certain pump intensity called the ASE threshold. A detailed characterization of the influence on the ASE performance of parameters such as the type of polymer and dyes used, the dye doping ratio, the thickness of the active film and the waveguide properties, has been previously carried out in PDI-doped polymer films. ${ }^{26-29}$ Such studies have been very useful to optimize the active material before the inclusion of the laser cavity. Among various PDIs investigated, the one denoted as PDI-C6 [N,N'-di(1hexylheptyl)perylene-3,4:9,10-tetracarboxylic diimide], showed an excellent ASE performance when dispersed at $0.5 \mathrm{wt} \%$ in PS films deposited over fused silica (for details on the chemical structure and conformation of PDI-C6 see ref. [29]). For this type of materials, it was shown that the ASE threshold depends on the thickness of the active film $\left(h_{\mathrm{f}}\right)$ and the behavior was explained in terms of the absorption, PL emission, number of waveguide modes and their confinement. ${ }^{28}$ The lowest thresholds were obtained for films supporting one highly confined mode, i.e. with $h_{\mathrm{f}}$ around $500-600 \mathrm{~nm}$. Such $h_{\mathrm{f}}$ value was chosen to prepare efficient single mode DFB lasers based on this type of waveguides..$^{7-9,29}$ The investigation of the ASE threshold 
dependence on film thickness has also been investigated in semiconducting polymer waveguides by other authors. ${ }^{30}$ Also in that case a correlation between the ASE threshold and the confinement of the fundamental waveguide mode was found. However, it should be noted that for these materials the intensity of the excitation beam decreases rapidly with depth due to the large film absorption, so the behavior for thick films might differ from that of low absorbing films such as the ones used in the present study.

In this work 1D second-order DFB lasers based on active laser films of PS doped with 0.5 wt. \% of PDI-C6, deposited over DCG layers with relief gratings recorded by HL, are reported. The advantage of using the photoresist layer directly as the substrate is that device fabrication is simplified. In order to reduce the expected high propagation losses of these structures, due to the low contrast between the refractive index of the PS film $\left(n_{\mathrm{f}}=1.59\right)$ and the substrate $\left(n_{\mathrm{DCG}}=1.55\right)$, which would lead to high laser thresholds, ${ }^{8,28}$ the thickness of both, the active film and the DCG layer, have been carefully adjusted. Following these guidelines, efficient DFB lasers with solvent resistant gratings, and therefore reusable, are demonstrated.

\section{SAMPLE PREPARATION AND EXPERIMENTAL PROCEDURES}

DFB laser fabrication consisted of the following steps: (1) Photoresist deposition: DCG layers of thickness 190 or $600 \mathrm{~nm}$ were deposited by spin-coating hot water solutions $\left(40^{\circ} \mathrm{C}\right)$ containing 4 and $10 \mathrm{wt} \%$ of DCG respectively, over fused silica substrates; (2) Grating recording: A relief grating was recorded over the DCG layer by using an Argon laser operating at $364 \mathrm{~nm}$. The pattern recorded was obtained by the interference of the direct beam with the beam reflected in a mirror attached to the sample holder. A scheme of the experimental setup used for grating recording can be found elsewhere. ${ }^{11}$ An absorbent glass was attached to the back side of the plate with an index matching liquid to avoid backward reflections from the surface of the 
glass support. The geometrical parameters for grating recording were adjusted in order to obtain different $\Lambda$ values in the range 367-381 nm ( $\pm 2 \mathrm{~nm})$; (3) Development: After desensitizing in a cool water bath $\left(15^{\circ} \mathrm{C}\right)$, relief gratings in the DCG film were obtained by a dry development in an oxygen electron cyclotron resonance (ECR) stream. Development times of 2 and 3.5 min were used to obtain grating depths of $50 \mathrm{~nm}$ and $80 \mathrm{~nm}( \pm 10 \mathrm{~nm})$, respectively. The gratings were characterized by field emission scanning electron microscopy (FE-SEM, ZEISS Ultra Plus) and atomic force microscopy (AFM, NT-MDT Solver PRO); (4) The active films were spin-coated over the DCG layers with gratings from toluene solutions containing PS as inert polymer and 0.5 wt. \% (with respect to PS) of PDI-C6. Film thickness (measured with an interferometer coupled to an optical microscope) was varied between 600 and $1100 \mathrm{~nm}$, by adjusting the percentage of PS with respect to the solvent (between 2.5 and 6 wt. \%). In order to characterize the ASE properties of the active materials, we also deposited films of similar characteristics on top of DCG layers without gratings, previously coated over fused silica substrates. These DCG layers were exposed to uniform illumination and processed following the same procedure described for obtaining the gratings, so the material parameters of the DCG were the same.

Since the concentration of PDI-C6 is very small, the refractive index of the active film $\left(n_{\mathrm{f}}\right)$ was calculated from the dispersion relation of un-doped PS films, given by $n_{\mathrm{f}}=A+\left(B / \lambda^{2}\right)+\left(C / \lambda^{4}\right)$, with $A=1.5691, B=0.0052, C=0.0007$ and $\lambda$ expressed in microns. ${ }^{28}$ Concerning the refractive index of the DCG layer $\left(n_{\mathrm{DCG}}\right)$, it was obtained from $n_{D C G}^{2}=1+\left[A_{1} \lambda^{2} /\left(\lambda^{2}-C_{1}^{2}\right)\right]$, with $\mathrm{A}_{1}=1.3420, \mathrm{C}_{1}=0.1250$ and $\lambda$ expressed in microns. This latter dispersion relation was experimentally determined by fitting data measured by the m-line technique $^{31}$ at various wavelengths, provided by a mercury lamp. Particularly, at $\lambda=579.5 \mathrm{~nm}$ (wavelength at which ASE appears for films thicker than $400 \mathrm{~nm}$ ), $n_{\mathrm{f}}=1.59$ and $n_{\text {DCG }}=1.55$. 
The waveguiding properties of the films were both, measured and modeled. Experimental $n_{\text {eff }}$ values associated with the transverse electric (TE) modes propagating in the structure were determined by the m-line technique at $\lambda=578 \mathrm{~nm}$. Theoretical $n_{\text {eff }}$ values were calculated by solving the propagation wave equation, following well known methods. ${ }^{32,33}$

The ASE properties of the films deposited over DCG without gratings were explored by optical excitation with a frequency-doubled Nd:YAG (YAG-yttrium aluminum garnet) laser (10 ns, $10 \mathrm{~Hz}$ ) operating at $532 \mathrm{~nm}$, which lies close to the maximum absorbance of PDI-C6. ${ }^{28} \mathrm{~A}$ scheme of the experimental setup can be found elsewhere. ${ }^{34}$ The energy of the pulses was varied using neutral density filters. A cylindrical lens and an adjustable slit were then used to shape the beam into a stripe of $3.5 \mathrm{~mm}$ by $0.53 \mathrm{~mm}$. The stripe was placed right up to the edge of the film, from were PL emission was collected with an Ocean Optics USB2000-UV-VIS fiber spectrometer. Spectral resolution was $1.3 \mathrm{~nm}$, so the error in measuring the emission linewith and wavelength were around $( \pm 0.8) \mathrm{nm}$ and $( \pm 0.4) \mathrm{nm}$, respectively.

The experimental setup to characterize the laser emission properties of the DFB devices differs from the one used to measure ASE only in the geometrical configuration to excite the sample and to collect the emitted light. In particular, the cylindrical lens was replaced by a spherical one, so the beam over the sample (incident at $\sim 20^{\circ}$ with respect to the normal to the film plane) was shaped into an elliptical spot (instead of a stripe) with a minor diameter of 1.1 $\mathrm{mm}$. The emitted light was collected in a direction perpendicular to the film surface (instead of from the edge), by placing the fiber spectrometer at $1 \mathrm{~cm}$ from the sample.

The operational device lifetime (i.e. the photostability) was determined by studying the time evolution of the laser intensity, while the sample was excited in the same region, at constant pump intensity and under ambient conditions. The parameter used to characterize this property 
has been the same used in previous works from our group, ${ }^{7,35}$ i.e. the photostability halflife $\left(\tau_{1 / 2}\right)$, defined as the time (or number of pump pulses) at which the laser intensity decays to half of its initial value.

\section{RESULTS AND DISCUSSION}

Results have been organized in three sections. Given the importance of characterizing the ASE properties of the active films before fabricating the DFB devices, we have first prepared PDI-C6doped PS films over DCG layers without gratings and studied the influence of changing film thickness. Results obtained are shown in section III.A, which have been interpreted in terms of the waveguide properties of the films, which have been both, measured and modeled. In section III.B we describe the results obtained with regards to the fabrication of DFB devices, which are designed taking into account the results obtained in section III.A. In section III.C we have prepared DFB devices with thinner DCG layers, aiming to further reduce the laser thresholds. Also in this section we provide information about the device operational lifetime. Finally, results are compared to those of DFB lasers based on the same active material, but with gratings engraved by NIL over $\mathrm{SiO}_{2}$ substrates, which were previously reported. ${ }^{7,8}$

\section{A. PDI-C6 doped PS films deposited over uniform (without gratings) DCG layers: ASE properties, waveguide characterization and modeling.}

In the introduction we already enhanced the importance of properly adjust the thickness of the active film $\left(h_{\mathrm{f}}\right)$ of a given waveguide structure, in order to obtain the lowest possible threshold. So, we first investigated the ASE properties of films of various thickness deposited over DCG layers without gratings. The thickness of the DCG layer $\left(h_{\mathrm{DCG}}\right)$ used in these 
experiments was $600 \mathrm{~nm}$. It is important to note that in the present case the difference between the refractive index of the active film $\left(n_{\mathrm{PS}}=1.59\right)$ and that of the gelatine substrate $\left(n_{\mathrm{DCG}}=1.55\right)$ is very small. So, in order to have a good confinement of the fundamental waveguide mode, a thickness larger than the optimal thickness of about 500-600 nm for the film deposited over fused silica, would be needed. Indeed, in the frame of waveguide theory, and considering the simplest possible model, in which the PDI-C6-doped PS film would be the guiding layer (with thickness $h_{\mathrm{g}}=h_{\mathrm{f}}$ ) deposited over a DCG substrate of infinite thickness, the thickness needed to have only the fundamental mode with maximum confinement would be around $1100 \mathrm{~nm}$ (just below the cut-off thickness for the appearance of the $\mathrm{TE}_{1}$ mode, i.e. $\left.1160 \mathrm{~nm}\right){ }^{36}$ Fig. 1 shows the emission linewidth (defined as the full width at half of the maximum, FWHM) as a function of the pump power density for films of various $h_{\mathrm{f}}$ values. As expected, the threshold ( $\left.I_{\text {th-ASE }}\right)$, defined as the pump intensity at which the FWHM decays to half of its maximum value, for the $1100 \mathrm{~nm}$-thick film is lower than the one of the film with $h_{\mathrm{f}}=600 \mathrm{~nm}$. Concerning the ASE emission wavelength and linewidth, the obtained values $\left(\lambda_{\mathrm{ASE}}=579.5 \mathrm{~nm}\right.$ and $\mathrm{FWHM}_{\mathrm{ASE}}=6$ $\mathrm{nm}$ ) are similar to those measured when the films are deposited over fused silica (see inset of Fig. 1). ${ }^{28}$

\section{INSERT FIG. 1}

In order to ensure that the threshold differences encountered were due to the confinement of the waveguide mode, the waveguiding properties have been investigated, both experimentally and theoretically for the films with $h_{\mathrm{f}}$ values of 600 and $1100 \mathrm{~nm}$, deposited over DCG. Characterization by the m-line technique of these two films showed the presence of two and three TE waveguide modes, respectively. This result was in disagreement with calculations based on the simple model previously mentioned, which predicted only one waveguide mode for both 
films. ${ }^{36}$ These observations pointed out to the idea that the effective thickness of the guiding layer should be larger than $h_{\mathrm{f}}$, which was the value assumed in these simple calculations. The inadequacy of using this simple model can be better understood by analyzing the electric field profile of the waveguide modes (see Fig. 2). As already mentioned, in these calculations it was assumed that the thickness of the DCG layer (considered as the substrate) was infinite, although in fact it is $600 \mathrm{~nm}$, and as observed in Fig. 2, light penetrates into the DCG further than this distance. In the case of higher order modes, the amount of light travelling along the DCG layer would be even larger, given that their electric field amplitude is maxima close to the interfaces. ${ }^{28}$ Note that the profiles for high order modes for these particular structures cannot be calculated in the same way as was done for the $\mathrm{TE}_{0}$ mode, because $h_{\mathrm{f}}$ is below the cutoff thickness for their propagation.

\section{INSERT FIGURE 2}

Taking into account these considerations we propose a simple model in which the fused silica would act as substrate, so $n_{\mathrm{s}}=n_{\mathrm{SiO} 2}$ and $h_{\mathrm{s}}=\infty$, and the guiding layer would be a film of effective thickness $h_{\mathrm{g}}=h_{\mathrm{f}}+h_{\mathrm{DCG}}$ and refractive index $n_{\mathrm{g}}$, being this latter value a weighted average of those of PS and DCG. Under these assumptions, the effective index of the structures can be easily calculated from the propagation wave equation as in the simplest model, but using these redefined $n_{\mathrm{s}}, h_{\mathrm{g}}$ and $n_{\mathrm{g}}$.

Given that the contribution of each layer depends on the type of mode and on the thickness of the layers, we propose the following expression to obtain a weighted average $n_{\mathrm{g}}$

$$
n_{g}=\left(h_{f} n_{f} w_{f}+h_{D C G} n_{D C G} w_{D C G}\right) /\left(h_{f} w_{f}+h_{D C G} w_{D C G}\right)
$$

where the parameters $w_{f}$ and $w_{D C G}$ are weight proportions assigned to the PS and DCG films, respectively, which represent the relative amount of light travelling along each of these layers. 
For $\mathrm{TE}_{0}$ modes, these have been calculated from the electric field profiles shown in Fig. 2, using the following equations: ${ }^{31,32}$

$$
w_{f}=\int_{0}^{h_{f}}|E(x)|^{2} d x / \int_{-\infty}^{h_{f}+h_{D C G}}|E(x)|^{2} d x ; \quad w_{D C G}=\int_{h_{f}}^{h_{f}+h_{D C G}}|E(x)|^{2} d x / \int_{-\infty}^{h_{f}+h_{D C G}}|E(x)|^{2} d x
$$

For the case of $\mathrm{TE}_{1}$ and $\mathrm{TE}_{2}$ modes, $w_{f}$ and $w_{D C G}$ have been estimated to be equal to 0.5 . This approximation is reasonable given that variations in these parameters in amounts of \pm 0.2 produced changes in the calculated $n_{\text {eff }}$ values, which were within the uncertainty range of the $n_{\text {eff }}$ experimentally measured. Fig. 3 displays the calculated $n_{\text {eff }}$ values for this model structure, whose scheme has been included as an inset in the same figure, as well as the experimental $n_{\text {eff }}$ values measured with the m-line technique. As observed, predictions and experimental results are in good agreement. The reason why the highest order modes predicted theoretically are not experimentally observed is because they are very close to the cutoff condition for their propagation.

\section{INSERT FIG. 3}

Going back to the results shown in Fig. 1 and considering previous discussions about the waveguide properties, it is possible to explain why the ASE threshold observed for the $1100 \mathrm{~nm}-$ thick PS film deposited over DCG is lower than that of the $600 \mathrm{~nm}$-thick film. Despite both films support high order modes, most of the light waveguided along the active film travels in the $\mathrm{TE}_{0}$ mode. As shown in Fig. 2 the confinement of this mode in the PS film is much larger for $h_{\mathrm{f}}=$ $1100 \mathrm{~nm}$ than for $h_{\mathrm{f}}=600 \mathrm{~nm}$. So losses, and consequently the threshold, will be lower in the former case. In accordance with results obtained with waveguides based on PS deposited over fused silica, ${ }^{28}$ as well as with semiconducting polymers, ${ }^{30}$ the influence on the threshold of the presence of higher order modes seems to be negligible. Note that the number of high order modes is larger for the film with $h_{\mathrm{f}}=1100 \mathrm{~nm}$ (two modes, $\mathrm{TE}_{1}$ and $\mathrm{TE}_{2}$ ), whose threshold is 
lower than that of the film with $h_{\mathrm{f}}=600 \mathrm{~nm}$, which supports only one (the $\mathrm{TE}_{1}$ ). The proposed model serves also to explain results obtained for other $h_{\mathrm{f}}$ values, particularly for $h_{\mathrm{f}}=950 \mathrm{~nm}$ (see Fig. 1) and $h_{\mathrm{f}}=1950 \mathrm{~nm}$ (for clarity, these data have not been displayed in Fig. 1 since they were similar to those of the film with $h_{\mathrm{f}}=1100 \mathrm{~nm}$ ). As observed, the threshold for the former film is between those of the $600 \mathrm{~nm}$ and $1100 \mathrm{~nm}$ thick-films previously measured, confirming the trend of lower threshold as the confinement of the fundamental mode improves, due to the increased film thickness. On the other hand, the threshold for the thickest film $(1950 \mathrm{~nm})$ was similar to that of the $1100 \mathrm{~nm}$-thick one, thus confirming the small influence on the ASE threshold of the presence of high order modes found in previous studies. ${ }^{28,30}$

In view of results obtained in this section, it can be concluded that for this type of waveguides with $600 \mathrm{~nm}$-thick DCG layers, the lowest ASE thresholds are obtained for PDIdoped PS thickness of around $1100 \mathrm{~nm}$ and above. So, this has been the chosen thickness to prepare DFB devices in section B.

\section{B. DFB lasers based on active PDI-C6-doped PS films deposited over DCG layers with gratings: Tuneability of the emission wavelength by grating period variation.}

DFB lasers were prepared by depositing 1100 nm-thick PDI-C6-doped PS films over DCG layers $\left(h_{\mathrm{DCG}}=600 \mathrm{~nm}\right)$ with gratings of $d \sim 80 \mathrm{~nm}$ and three different periods $\left(\Lambda_{1}=367\right.$ $\mathrm{nm}, \Lambda_{2}=378$ and $\Lambda_{3}=381 \mathrm{~nm}$ ), previously recorded by HL. A scheme of the DFB laser device is depicted in Fig. 4(a), while Figs. 4(b) and 4(c) show SEM and AFM images, respectively, of one of the gratings over the DCG layer, before the deposition of the PDI-C6-doped PS film. The SEM photograph shows that the grating lines present many irregularities, caused by the proximity of the grating period to the resolution limit of the DCG. The DCG layer is composed 
of fibers with a diameter of around $50 \mathrm{~nm}$, randomly distributed but oriented rather parallel to the substrate surface. ${ }^{37}$ Nevertheless, it is observed that the grating structure is clear and the period is well defined. The AFM image shows a profile with a groove width to period ratio of about $1 / 5$ and a groove depth of approximately $80 \mathrm{~nm}$. It is remarkable that these gratings over DCG are highly resistant to organic solvents, so they can be used multiple times (i.e. the active layers can be eliminated with organic solvents and then, new films can be deposited on top).

\section{INSERT FIG. 4}

The use of gratings with different periods allowed tuning the laser wavelength $\left(\lambda_{\mathrm{DFB}}\right)$. This is illustrated in Fig. 5(a), which shows the emission spectra (above threshold) of the various lasers prepared. The ASE spectrum of a similar film deposited over a DCG layer without grating has also been included in the figure. The DFB threshold $\left(I_{\mathrm{th}-\mathrm{DFB}}\right)$ for a given device was determined from the plot of its emission intensity at $\lambda_{\text {DFB }}$ versus the pump intensity (see Fig. 5(b)). In particular, $I_{\text {th-DFB }}$ corresponds to the pump intensity at which a drastic increase in the emission intensity is observed. The $\lambda_{\mathrm{DFB}}$ and $I_{\mathrm{th}-\mathrm{DFB}}$ parameters for the three lasers are listed in Table I.

\section{INSERT FIG. 5}

\section{INSERT TABLE I}

DFB emission for all the devices consisted in a single peak, although as described in section III.A., the waveguides support three modes. In a previous work about DFB lasers based on PDI-C6-doped PS films deposited over gratings engraved on $\mathrm{SiO}_{2}$ by NIL, ${ }^{8}$ it was shown that for film thickness larger than around $1000 \mathrm{~nm}$, DFB emission appeared at two different wavelengths that corresponded to the two waveguide modes propagating in the structure. The 
thresholds for each peak were generally different, so it was found that under intense pumping conditions one of the peaks dominated. In accordance with those results, in the present case we would expect to see emission at three different wavelengths. In contrast, only one peak is observed, which presumably would correspond to the one with lowest threshold. By using the $n_{\text {eff }}$ values determined experimentally by the m-line technique for the various waveguide modes

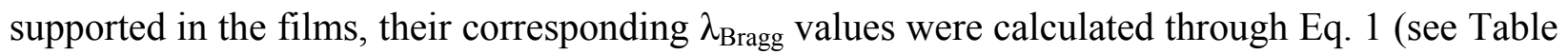
I). By comparing these results with the experimental $\lambda_{\mathrm{DFB}}$ values (obtained from the emission spectra), it is possible to determine the waveguide mode associated to each DFB peak. The device with lowest threshold is the one with $\Lambda=367 \mathrm{~nm}\left(\Lambda_{1}\right.$ in Fig. 5), whose $\lambda_{\text {DFB }}$ is very close to the maximum of the gain spectrum (i.e. $\lambda_{\mathrm{ASE}}$ ) and which corresponds to the $\mathrm{TE}_{0}$ mode. The laser with $\Lambda=378 \mathrm{~nm}\left(\Lambda_{2}\right)$ emits also very close to $\lambda_{\mathrm{ASE}}$, so the reason why its threshold is higher than that of the previous one appears to be the fact that in this case the associated mode is the $\mathrm{TE}_{1}$. Finally, the laser with $\Lambda=381 \mathrm{~nm}\left(\Lambda_{3}\right)$, associated to the $\mathrm{TE}_{1}$, shows the highest threshold, mainly because it emits much further away from $\lambda_{\mathrm{ASE}}$ than the others. These results are in agreement with other works published in the literature which concluded that the most important parameter determining the DFB laser threshold is the proximity to the maximum of the gain spectrum..$^{8,9,38-40}$ The type of mode has also some influence on the threshold, which is generally lower for $\mathrm{TE}_{0}$ due to its lower propagation losses. ${ }^{8-28}$ However, its influence is less significant, so in order to be appreciated, the emission wavelength of the devices under comparison should be similar.

\section{DFB threshold optimization by adjusting the thickness of the DCG layer and photostability characterization}


As discussed in previous sections, light penetrates considerably into the DCG layer up to the point that it is the $\mathrm{SiO}_{2}$ the one that acts as substrate, so light is waveguided along both, the PS and the DCG films. This prompted us to think about the possibility of reducing the propagation losses, and therefore the DFB threshold, by reducing the thickness of the DCG layer. Given that the DCG film does not contain active PDI molecules, this would not affect the gain properties.

DFB gratings with a depth of around $50 \mathrm{~nm}$ and different periods $\left(\Lambda_{4}=356 \mathrm{~nm}, \Lambda_{5}=376\right.$ $\mathrm{nm}$ and $\Lambda_{6}=391 \mathrm{~nm}$ ) were recorded over DCG films with $h_{\mathrm{DCG}}=190 \mathrm{~nm}$, previously deposited over $\mathrm{SiO}_{2}$ substrates. This particular $h_{\mathrm{DCG}}$ value was chosen in order to have a ratio between film thickness and grating depth similar to the one used in devices prepared in section $\mathrm{B}$, as well as to ensure sufficient reproducibility and uniformity in film thickness and grating profile. Device fabrication was completed by spin-coating a PDI-C6-doped PS film. In this case $600 \mathrm{~nm}$-thick PS films were used, instead of the $1100 \mathrm{~nm}$-thick ones employed in devices of section B. According to the proposed waveguide model, the fused silica acts as substrate and in the present case the influence of the DCG layer will be smaller due to its reduced thickness. Therefore, in contrast with the case of lasers based on thick DCG layers, in which the confinement of the fundamental waveguide mode was better for the film with $h_{\mathrm{f}}=1100 \mathrm{~nm}$, in the present case, mode confinement would be similar for both film thickness. An advantage of using $600 \mathrm{~nm}$-thick films in this case is that the number of higher order modes will be smaller. Although as already discussed, the presence of high order modes does not seem to affect significantly the ASE thresholds, reducing its number in a given structure allows having a better control of the particular waveguide mode at which DFB emission takes place. In any case, at the end of this 
section, a DFB device with $h_{\mathrm{f}}=1100 \mathrm{~nm}$ was prepared, in order to verify the convenience of using in this case $h_{\mathrm{f}}=600 \mathrm{~nm}$.

The DFB spectra of the devices are shown in Fig. 6 . The corresponding $\lambda_{\mathrm{DFB}}$ and $I_{\mathrm{th}-\mathrm{DFB}}$

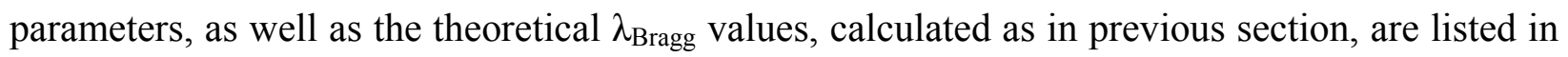
Table II. Also in this case DFB emission appears at a wavelength close to one of the waveguide modes. Particularly to the one whose wavelength is closer to $\lambda_{\text {ASE }}$. In accordance with results obtained in section III.B., the device whose emission wavelength is the closest one to $\lambda_{\mathrm{ASE}}$ [with $\Lambda=376 \mathrm{~nm}\left(\Lambda_{5}\right)$ ], has shown the lowest threshold (10 $\mu \mathrm{J} /$ pulse $)$ among the three devices, which in addition is associated to the $\mathrm{TE}_{0}$ mode. It is remarkable that this threshold is also lower that the one obtained for the best performing device with thicker DCG layer [with $\Lambda=367 \mathrm{~nm}\left(\Lambda_{1}\right)$, see Table I], whose emission was also very close to $\lambda_{\mathrm{ASE}}$ and corresponded to the $\mathrm{TE}_{0}$ mode. As previously discussed, a device with a thick active film $\left(h_{\mathrm{f}}=1100 \mathrm{~nm}\right)$ and a thin DCG layer $\left(h_{\mathrm{DCG}}=190 \mathrm{~nm}\right)$ was also fabricated and characterized. The results have been included in Table II. The emission wavelength of this laser is very similar to that of the one with the same parameters but with thinner thickness, and is very close to $\lambda_{\mathrm{ASE}}$. This is possible because the associated mode in this case is the $\mathrm{TE}_{1}$, instead of $\mathrm{TE}_{0}$. This result indicates that performing a deep analysis of the modes propagating in the DFB laser is a key issue in order to design tuneable devices by film thickness variation. With regards to the threshold, it is slightly higher (13 $\mu \mathrm{J} /$ pulse) for the device with the thicker active film, which can be explained by the fact that the associated mode is the $\mathrm{TE}_{1}$. Finally, it is also remarkable that despite this increase in threshold, it is still lower than the one obtained in the previous section with the thicker DCG layer. These results demonstrate our success in improving the performance by decreasing the thickness of the DCG layer, although this aspect leaves still room to further improvement. 
Ideally, it would be desirable to reduce $h_{\mathrm{DCG}}$ as much as possible, firstly to diminish the losses, but more importantly, to avoid the presence of high order waveguide modes. As previously shown, the presence of various waveguide modes complicates a precise control when aiming to tune the DFB wavelength by changing the grating period. If various modes are present, DFB emission can occur at any of them, particularly at the one whose wavelength is the closest to that of maximum gain (i.e. the ASE wavelength). Progress in this direction would require further work that is out of the scope of the present study. The challenge would be to obtain very thin films and very shallow gratings, while keeping good film uniformity and grating quality, sufficiently large gain, as well as index contrast, so high PL efficiency, low ASE and DFB thresholds can be achieved.

\section{INSERT FIG. 6}

\section{INSERT TABLE II}

It is also important to compare the threshold of these devices to those of lasers based on the same type of active films deposited over $\mathrm{SiO}_{2}$ with gratings fabricated by NIL. In ref. [8] a wide set of such type of lasers, with film thickness varying between 240 and $1200 \mathrm{~nm}$ and grating depths between 30 and $240 \mathrm{~nm}$, were reported. It was found that for devices with any of the grating depths studied and with films of thickness in the range $660-775 \mathrm{~nm}$, DFB emission corresponded to the $\mathrm{TE}_{0}$ mode, it appeared very close to $\lambda_{\mathrm{ASE}}$ (in the interval $575-579 \mathrm{~nm}$ ) and its threshold was similar for all devices (around $2 \mu \mathrm{J} /$ pulse). This threshold value is only five times smaller than the one found for the best performing device prepared here. Taking into account that the quality of the gratings recorded by LH in our devices (see Figs. 4(b) and 4(c)) is not as good as the one achieved by NIL, besides the different type of profile (sinusoidal versus square), we can conclude that lasers prepared in this work have a very good performance. 
The laser operational lifetime was measured as described in the experimental section. For example, for the device with $h_{\mathrm{f}}=1100 \mathrm{~nm}$ and $h_{\mathrm{DCG}}=190 \mathrm{~nm}$, a photostability halflife of $\tau_{1 / 2}=$ 55 min (i.e. 33000 pump pulses) was obtained when excitation was performed at a pump energy of $30 \mu \mathrm{J} /$ pulse $\left(281 \mathrm{~kW} / \mathrm{cm}^{2}\right)$. This value is comparable to that reported for DFB devices with gratings engraved by $\mathrm{NIL}$ on $\mathrm{SiO}_{2}$ substrates and based on the same active material, when pumped at the similar pump power densities. ${ }^{7}$ As discussed in detailed previously, the power density delivered over the sample seems to be the most important parameter determining the photostability halflife for these materials. ${ }^{7,35}$

\section{CONCLUSIONS}

Second-order distributed feedback (DFB) lasers based on perylenediimide-doped polystyrene (PS) films, deposited over relief gratings recorded by holographic lithography (HL) on dichromated gelatine (DCG) photoresist layers, have been fabricated and characterized. A distinct characteristic of these lasers is that the photoresist layer with the grating is directly used as the substrate of the device. This allows simplifying the fabrication process and the grating pattern is better preserved since no etching methods are needed. In addition, the obtained gratings are highly resistant to organic solvents, so they can be reused multiple times. The experimentally measured waveguide properties (number and type of modes and their corresponding effective indexes) have been successfully interpreted by means of a simple model in which light is waveguided along both, the PS and the DCG films. This model has allowed identifying the waveguide mode at which DFB emission appears, which has been crucial to optimize the laser thresholds and to control their wavelength tuneability. A key point to minimize the laser threshold has been to choose the proper PS and DCG thickness, so DFB emission corresponds to the fundamental transverse electric mode $\left(\mathrm{TE}_{0}\right)$ and it appears as closest 
as possible to the wavelength of maximum gain (i.e., that at which amplified spontaneous emission occurs in films without gratings). Reducing the thickness of the DCG film has also allowed decreasing the threshold, which is attributed to a reduction of the propagation losses. The performance of these lasers is comparable (similar operational lifetimes and thresholds only a few times smaller) to that of recently reported devices based on the same active material, but deposited over high-quality DFB gratings engraved on $\mathrm{SiO}_{2}$ by nanoimprint lithography.

\section{ACKNOWLEDGMENTS}

We thank support from the Spanish Government (MINECO) and the European Community (FEDER) through grants MAT2008-06648-C02 and MAT-2011-28167-C02, as well as to the University of Alicante. MGR is supported by a CSIC fellowship within the program JAE. We also acknowledge V. Esteve for technical assistance. 


\section{References}

1 M.D. McGehee and A.J. Heeger, Adv. Mater. 12, 1655 (2000).

2 I.D.W. Samuel and G.A. Turnbull, Chem. Rev. 107, 1272 (2007).

3 S. Chenais and S. Forget, Polym. Int. 61, 390 (2011).

$4 \quad$ Y. Yang, G.A. Turnbull and I.D.W. Samuel, Appl. Phys. Lett. 92, 163306 (2008).

$5 \quad$ J. Clark and G. Lanzani, Nature Photon. 4, 438 (2010).

$6 \quad$ L.J. Guo, Adv. Mater. 19, 495 (2007).

7 V. Navarro-Fuster, E.M. Calzado, P.G. Boj, J.A. Quintana, J.M. Villalvilla, M.A. Díaz-García, V. Trabadelo, A. Juarros, A. Retolaza and S. Merino, Appl. Phys. Lett. 97, 171104 (2010). V. Navarro-Fuster, I. Vragovic, E.M. Calzado, P.G. Boj, J.A. Quintana, J.M. Villalvilla, A. Retolaza, A. Juarros, D. Otaduy, S. Merino and M.A. Díaz-García, J. Appl. Phys. 112, 043104 (2012).

9 M.G. Ramirez, P.G. Boj, V. Navarro-Fuster, I. Vragovic, J.M. Villalvilla, I. Alonso, V. Trabadelo, S. Merino and M.A. Díaz-García, Opt. Express 19, 22443 (2011).

10 J. R. Lawrence, P. Andrew, W. L. Barnes, M. Buck, G. A. Turnbull and I.D.W. Samuel, Appl. Phys. Lett. 81, 1955 (2002).

11 E. M. Calzado, J.M. Villalvilla, P.G. Boj, J.A. Quintana, P.A. Postigo and M.A. Díaz-García, Appl. Opt. 49, 463 (2010).

12 L. Rocha, V. Dumarcher, C. Denis, P. Raimond, C. Fiorini, and J. M. Nunzi, J. Appl. Phys. 89, 3067 (2001).

13 J. Mysliwiec, L. Sznitko, A. Sobolewska, S. Bartkiewicz and A. Miniewicz, Appl. Phys. Lett. 96, 141106 (2010).

14 T. Ubukata, T. Isoshima and M. Hara, Adv. Mater. 17, 1630 (2005).

15 C. Ye, K. Y. Wong, Y. He and X. Wang, Opt. Express 15, 936 (2007).

16 L. M. Goldenberg, V. Lisinetskii, Y. Gritsai, J. Stumpe and S. Schrader, Adv. Mater. 24, 3339 (2012).

17 A. Mahfoud Familia and A. Sarangan, Opt. Commun. 281, 310 (2008).

18 M.B. Christiansen, T. Buß, C.L.C. Smith, S.R. Petersen, M.M. Jørgensen and A. Kristensen, J. Micromech. Microeng. 20, 115025 (2010).

19 M.M. Jørgensen, S.R. Petersen, M.B. Christiansen, T. Buß, C.L.C. Smith and A. Kristensen, Appl. Phys. Lett. 96, 231115 (2010). 
C. Vannahme, C. L.C. Smith, M.B. Christiansen and A. Kristensen, Appl. Phys. Lett. 101, 151123 (2012).

H. Kogelnik and C. V. Shank, Appl. Phys. Letters 18, 152 (1971).

D. Meyerhofer, Appl. Opt. 10, 416 (1971).

(1998).

\section{J. A. Quintana, P. G. Boj, J. Crespo, J. A. Vallés-Abarca and J. M. Villalvilla, Thin Solid Films 317, 343} (1998).

M.D. McGehee, R. Gupta, S. Veenstra, E.M. Miller, M.A. Díaz-García and A.J. Heeger, Phys. Rev. B 58, 7035 (1998).

E.M. Calzado, J.M. Villalvilla, P.G. Boj, J.A. Quintana, R. Gómez, J.L. Segura and M.A. Díaz-García, J. Phys. Chem. C 111, 13595 (2007).

E.M. Calzado, J.M. Villalvilla, P.G. Boj, J.A. Quintana, R. Gómez, J.L. Segura and M.A. Díaz-García, Appl. Opt. 46, 3836 (2007).

E.M. Calzado, M.G. Ramírez, P.G. Boj and M.A. Díaz García, Appl. Opt. 51, 3287 (2012).

M.G. Ramirez, M. Morales-Vidal, V. Navarro-Fuster, P.G. Boj, J.A. Quintana, J.M. Villalvilla, A. Retolaza, S. Merino and M.A. Díaz-García, J. Mater. Chem. C 1, 1182 (2013).

M. Anni, A. Perulli and G. Monti, J. Appl. Phys. 111, 093109 (2012).

A. Boudrioua, Photonic waveguides: theory and applications. (John Wiley \& Sons, New Jersey, 2009).

C.-L. Chen, Foundations of guided-wave optics. (John Wiley \& Sons, New Jersey, 2006).

E.M. Calzado, J.M. Villalvilla, P.G. Boj, J.A. Quintana and M.A. Díaz-García, J. Appl. Phys. 97, 093103 (2005).

M.A. Díaz-García, E.M. Calzado, J.M. Villalvilla, P.G. Boj, J.A. Quintana, F. Giacalone, J.L. Segura and N. Martín, J. Appl. Phys. 97, 063522 (2005).

E.M. Calzado, J.M. Villalvilla, P.G. Boj, J.A. Quintana, V. Navarro-Fuster, A. Retolaza, S. Merino and M.A. Díaz-García, Appl. Phys. Lett. 101, 223303 (2012).

J. Crespo, M. Pardo, M. A. Satorre and J. A. Quintana, Appl. Opt. 32, 3068 (1993).

R. Xia, G. Heliotis, P.N. Stavrinou and D.D.C. Bradley, Appl. Phys. Lett. 87, 031104 (2005). 
39 D. Schneider, T. Rabe, T. Riedl, T. Dobbertin, M. Kroger, E. Becker, H.H. Johannes, W. Kowalsky, T. Weimann, J. Wang and P. Hinze, Appl. Phys. Lett. 85, 1659 (2004).

40 S. Klinkhammer, T. Woggon, U. Geyer, C. Vannahme, S. Dehm, T. Mappes and U. Lemmer, Appl. Phys. B 97, 787 (2009). 


\section{Figure captions}

Figure 1. (Color on line) Emission linewidth as a function of pump intensity for PDI-C6-doped PS films deposited over $600 \mathrm{~nm}$-thick DCG layers without gratings (circles, stars and squares for film thickness $\left(h_{\mathrm{f}}\right)$ of 600, 950 and $1100 \mathrm{~nm}$, respectively) and over fused silica (triangles, $h_{\mathrm{f}}=$ $600 \mathrm{~nm}$ ); The inset shows the corresponding ASE spectra: films with $h_{\mathrm{f}}$ values of 600,950 and $1100 \mathrm{~nm}$ over DCG (dashed, dotted and full lines, respectively) and $h_{\mathrm{f}}=600 \mathrm{~nm}$ over fused silica (dash-dotted line).

Figure 2. (Color on line) Electric field profiles for $\mathrm{TE}_{0}$ modes, calculated from the propagation wave equation at $\lambda=579 \mathrm{~nm}$ for a waveguide of thickness $h_{\mathrm{g}}=h_{\mathrm{f}}$ (suffix $f$ refers to PDI-C6doped PS film) and refractive index $n_{\mathrm{g}}=n_{\mathrm{f}}$, deposited over a DCG substrate $\left(n_{\mathrm{s}}=n_{\mathrm{DCG}}\right.$ and $h_{\mathrm{s}}=$ $\infty)$

Figure 3. (Color on line) Effective refractive index $\left(n_{\mathrm{eff}}\right)$ for TE modes as a function of $h_{\mathrm{f}}+$ $h_{\mathrm{DCG}}$ : symbols are experimental $n_{\mathrm{eff}}$ values determined by the m-line technique at $\lambda=578 \mathrm{~nm}$ for $h_{\mathrm{f}}=600 \mathrm{~nm}$ (squares) and $h_{\mathrm{f}}=1100 \mathrm{~nm}$ (triangles) and full lines are calculations from the

propagation wave equation for a waveguide of thickness $h_{\mathrm{g}}=h_{\mathrm{f}}+h_{\mathrm{DCG}}\left(h_{\mathrm{DCG}}=600 \mathrm{~nm}\right.$ in all cases) and refractive index $n_{\mathrm{g}}$ (obtained from Eq. (2)) deposited over a fused silica substrate $\left(n_{\mathrm{s}}=\right.$ $n_{\mathrm{SiO} 2}$ and $\left.h_{\mathrm{s}}=\infty\right)$. 
Figure 4. (Color on line) (a) Scheme of the DFB device; (b) SEM image and (c) AFM profile of a relief grating with $\Lambda=378 \mathrm{~nm}$ and $\mathrm{d}=80 \mathrm{~nm}$, recorded over a DCG layer $\left(h_{\mathrm{DCG}}=600 \mathrm{~nm}\right)$ deposited over fused silica.

Figure 5. (Color on line) (a) Emission spectra (above threshold) of DFB lasers based on PDI-C6doped PS films $\left(h_{\mathrm{f}}=1100 \mathrm{~nm}\right)$ deposited on DCG layers $\left(h_{\mathrm{DCG}}=600 \mathrm{~nm}\right)$ with gratings of various periods $(\Lambda)$ and ASE spectrum of a similar film deposited over a uniform DCG layer; (b) Output intensity at $\lambda_{\mathrm{DFB}}$ for the DFB lasers whose data are displayed in (a).

Figure 6. (Color on line) Emission spectra (above threshold) of DFB lasers based on PDI-C6doped PS films $\left(h_{\mathrm{f}}=600 \mathrm{~nm}\right)$ deposited on DCG layers $\left(h_{\mathrm{DCG}}=190 \mathrm{~nm}\right)$ with gratings of various periods $(\Lambda)$ and ASE spectrum of a similar film deposited over a uniform DCG layer. 


\section{Tables}

TABLE I. Laser performance of DFB lasers based on PDI-C6-doped PS films $\left(h_{\mathrm{f}}=1100 \mathrm{~nm}\right)$ deposited on DCG layers $\left(h_{\mathrm{DCG}}=600 \mathrm{~nm}\right)$.

\begin{tabular}{|c|c|c|c|c|}
\hline$\Lambda^{\mathrm{a}}(\mathrm{nm})$ & Waveguide mode & $\lambda_{\text {Bragg }}^{\text {calc b }}(\mathrm{nm})$ & $\lambda_{\mathrm{DFB}}^{\exp \mathrm{c}}(\mathrm{nm})$ & $\begin{array}{c}I_{\text {th-DFB }}{ }^{\mathrm{d}} \\
\left(\mu \mathrm{J} \cdot \text { pulse }^{-1}\right)\end{array}$ \\
\hline \multirow[t]{3}{*}{1367} & $\mathbf{T E}_{0}$ & 583 & 579.4 & 16 \\
\hline & $\mathrm{TE}_{1}$ & 571 & & \\
\hline & $\mathrm{TE}_{2}$ & 554 & & \\
\hline \multirow[t]{3}{*}{${ }^{2} 378$} & $\mathrm{TE}_{0}$ & 600 & & \\
\hline & $\mathbf{T E}_{1}$ & 588 & 580.5 & 45 \\
\hline & $\mathrm{TE}_{2}$ & 570 & & \\
\hline \multirow[t]{3}{*}{${ }^{3} 381$} & $\mathrm{TE}_{0}$ & 605 & & \\
\hline & $\mathbf{T E}_{1}$ & 592 & 585.2 & 90 \\
\hline & $\mathrm{TE}_{2}$ & 575 & & \\
\hline
\end{tabular}

${ }^{\mathrm{a}} \Lambda$ is the period of DFB grating, where the superscript indicates the numeric notation used in Fig. 5 (error is $\pm 2 \mathrm{~nm}$ ).

${ }^{\mathrm{b}} \lambda_{\text {Bragg }}^{\text {calc }}$ is the Bragg wavelength (error is $\pm 6 \mathrm{~nm}$ ) calculated from Eq. (1) with $n_{\text {eff }}$ determined by the mline technique (data in Fig. 2).

${ }^{\mathrm{c}} \lambda_{\mathrm{DFB}}{ }^{\mathrm{exp}}$ is the experimental DFB emission wavelength (error is $\pm 0.4 \mathrm{~nm}$ ).

${ }^{\mathrm{d}} I_{\text {th-DFB }}$ is the experimental DFB threshold (error is $10 \%$, estimated as a standard deviation from measurements on different nominally identical samples). 
TABLE II. Laser performance of DFB lasers based on PDI-C6-doped PS films deposited on DCG layers $\left(h_{\mathrm{DCG}}=190 \mathrm{~nm}\right)$.

\begin{tabular}{|c|c|c|c|c|c|c|}
\hline$\Lambda^{a} / \mathrm{nm}$ & $h_{\mathrm{f}}^{b}(\mathrm{~nm})$ & $\begin{array}{c}\text { Waveguide } \\
\text { mode }\end{array}$ & $n_{\mathrm{eff}}^{c}$ & $\begin{array}{c}\lambda_{\text {Bragg }} \text { calc } d \\
\text { (nm) }\end{array}$ & $\begin{array}{c}\lambda_{\mathrm{DFB}} \exp e \\
(\mathrm{~nm})\end{array}$ & $\begin{array}{c}I_{\text {th-DFB }}{ }^{f} \\
\left(\mu \mathrm{J} \cdot \text { pulse }^{-1}\right)\end{array}$ \\
\hline \multirow[t]{2}{*}{${ }^{4} 356$} & 600 & $\mathrm{TE}_{0}$ & 1.57 & 560 & 568.7 & 100 \\
\hline & & $\mathrm{TE}_{1}$ & 1.48 & 528 & & \\
\hline \multirow[t]{2}{*}{${ }^{5} 376$} & 600 & $\mathrm{TE}_{0}$ & 1.57 & 592 & 580.0 & 10 \\
\hline & & $\mathrm{TE}_{1}$ & 1.48 & 558 & & \\
\hline \multirow[t]{2}{*}{${ }^{6} 391$} & 600 & $\mathrm{TE}_{0}$ & 1.57 & 615 & & \\
\hline & & $\mathrm{TE}_{1}$ & 1.48 & 580 & 587.4 & 570 \\
\hline \multirow[t]{3}{*}{${ }^{5} 376$} & 1100 & $\mathrm{TE}_{0}$ & 1.59 & 596 & & \\
\hline & & $\mathrm{TE}_{1}$ & 1.54 & 579 & 580.1 & 13 \\
\hline & & $\mathrm{TE}_{2}$ & 1.47 & 553 & & \\
\hline
\end{tabular}

${ }^{\mathrm{a}} \Lambda$ is the period of DFB grating, where the superscript indicates the numeric notation used in Fig. 6 (error is $\pm 2 \mathrm{~nm})$.

${ }^{\mathrm{b}} h_{\mathrm{f}}$ is the film thickness (error is $5 \%$ ).

${ }^{c} n_{\text {eff }}$ is the effective index determined by the m-line technique (error is $\pm 0.01 \mathrm{~nm}$ ).

${ }^{\mathrm{d}} \lambda_{\text {Bragg }}{ }^{\text {calc }}$ is the Bragg wavelength calculated from Eq. (1) with $n_{\text {eff }}$ values determined by the m-line technique (error is $\pm 6 \mathrm{~nm}$ ).

${ }^{\mathrm{e}} \lambda_{\mathrm{DFB}}{ }^{\mathrm{exp}}$ is the experimental DFB emission wavelength (error is $\pm 0.4 \mathrm{~nm}$ ).

${ }^{\mathrm{f}} I_{\text {th-DFB }}$ is the Experimental DFB threshold (error is $10 \%$, estimated as a standard deviation from measurements on different nominally identical samples). 


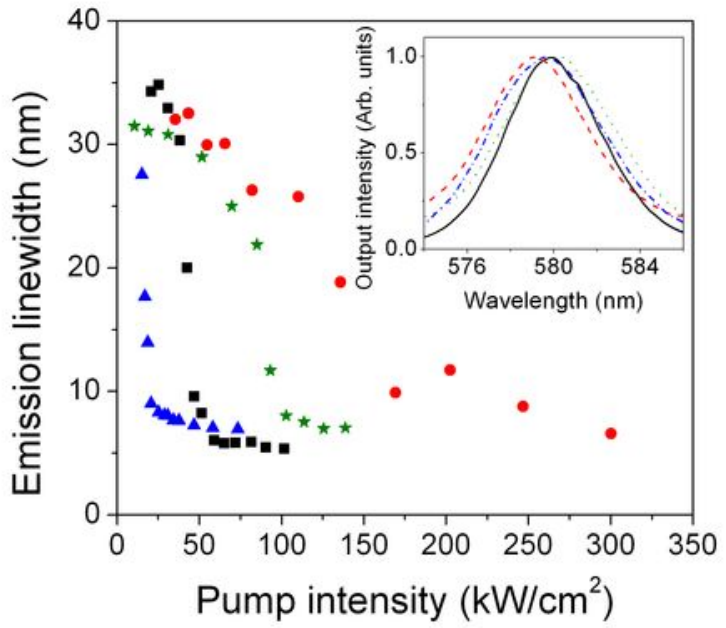




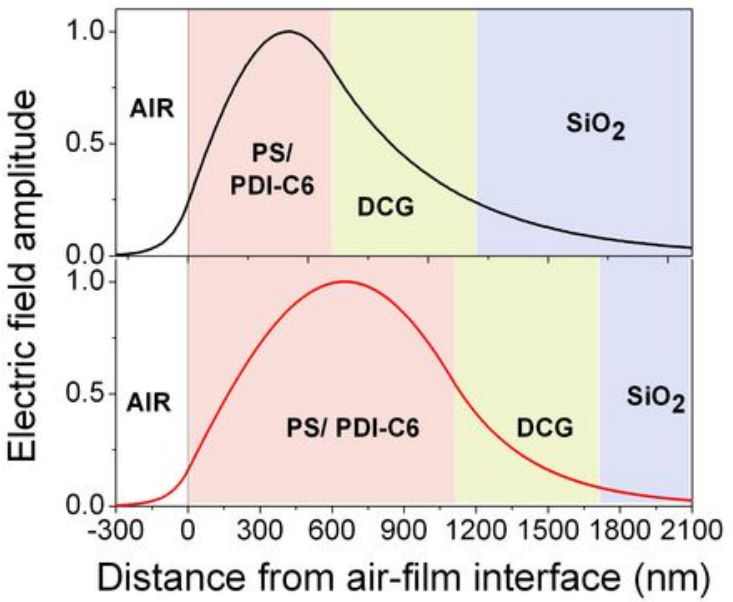




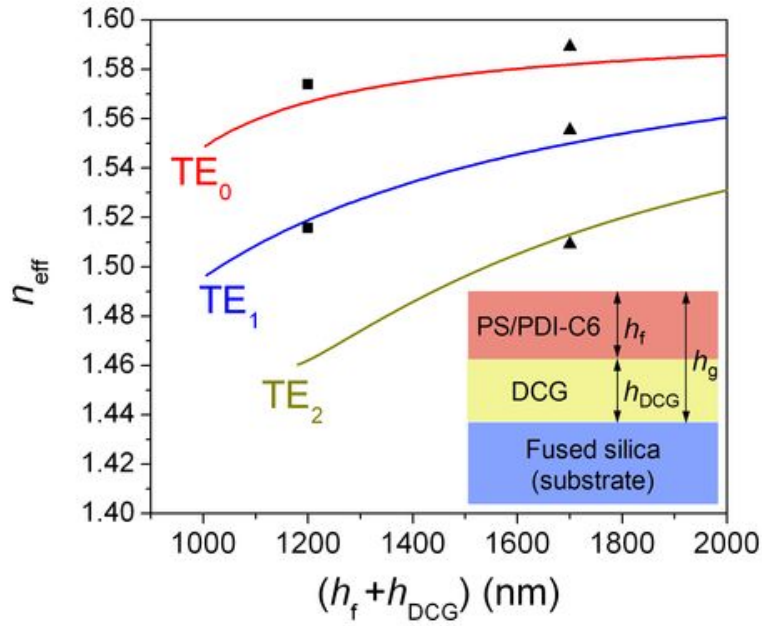



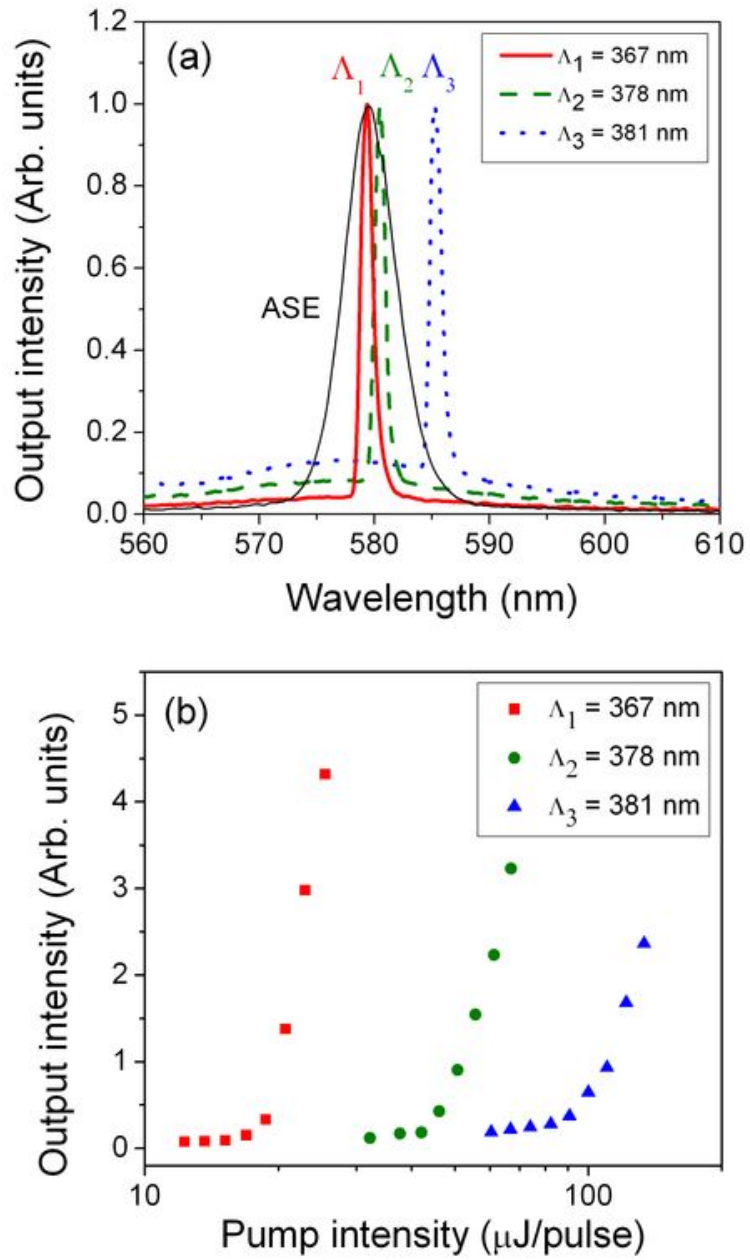


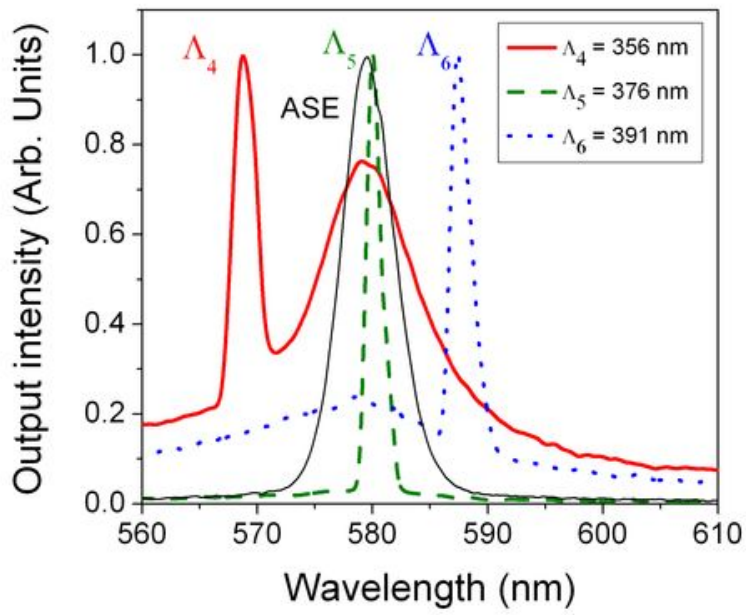

